\title{
A cross-lagged model of reinforcement sensitivity, personality and affectivity
}

\author{
Blaž Rebernjak ${ }^{A, B, C, D, E, F}$, Vesna Buško $o^{A, B, C, D, E, F, G}$ \\ Faculty of Social Sciences and Humanities, University of Zagreb, Zagreb, Croatia
}

\section{BACKGROUND}

There is a rich body of literature describing the links between behavioral activation/inhibition, personality and trait affectivity. The sensitivity of the behavioral activation system (BAS) is related to extraversion and positive affect, while the sensitivity of the behavioral inhibition is related to neuroticism and negative affect. Besides the strong links observed, it is unclear what the causal relationships between these constructs are.

\section{PARTICIPANTS AND PROCEDURE}

The aim of this study was to provide indirect support for the theoretical accounts postulating the causal direction from behavioral sensitivity through personality to affectivity. Using data from two measurement occasions, two sets of models were specified to test those predictions. A total of 286 social sciences and humanities students participated in the study for course credit or financial reimbursement.
RESULTS

Our results provide limited support for the proposed causal direction, but only some of the direct links were observed to be significantly different from zero. Extraversion and positive affect showed a reciprocal pattern of influences, while the sensitivity of the behavioral inhibition system (BIS) predicted neuroticism.

\section{CONCLUSIONS}

The results presented here give limited support to the proposed direction of relationships between the three sets of affective constructs investigated in this study. BIS and BAS sensitivities and personality measures showed higher stability in the one-month period compared to positive and negative affectivity.

\section{KEY WORDS}

behavioral activation/inhibition; personality; affectivity; RST

CORRESPonding AUthor - Blaž Rebernjak, Ph.D., Faculty of Social Sciences and Humanities, University of Zagreb,

I. Lučića 3, 100000 Zagreb, Croatia, e-mail: brebernj@ffzg.hr

AUthors' CONTRIBUtion - A: Study design - B: Data collection - C: Statistical analysis - D: Data interpretation .

E: Manuscript preparation · F: Literature search · G: Funds collection

TO CITE THIS ARTICLE - Rebernjak, B., \& Buško, V. (2017). A cross-lagged model of reinforcement sensitivity, personality

and affectivity. Current Issues in Personality Psychology, 5(2), 83-90.

RECEIVED 04.11.2016 · REVIEWED 26.01.2017 · ACCEPTED 21.02.2017 · PUBLISHED 11.05.2017 


\section{BACKGROUND}

Individual differences in behavior are explicitly linked to individual differences in the regulation and experience of emotions (Watson, 2000). More specifically, the behavioral tendencies to avoid potential harm or approach potential rewards can be explained by the constitution of the affective system. One interesting question that can be asked about this relation is: Do affective characteristics influence stable behavioral tendencies or does the personality set the tone for experiencing specific affective states? Furthermore, the causality could work in both directions - personality could predispose individuals to experience certain emotions, but also the experience of certain emotions could reflect on the stable behavioral tendencies.

There is a robust link between certain aspects of personality such as extraversion and neuroticism and the tendency to experience positive and negative emotions. Individuals with higher scores on measures of extraversion tend to exhibit more self-reported positive affect (PA). Put another way, people who are gregarious and sociable report more positive emotional states compared to people who are withdrawn and quiet (Watson \& Clark, 1997; Gable, Reis, \& Elliot, 2003; Wilt, Noftle, Fleeson, \& Spain, 2012). In a meta-analysis of 52 studies, Steel, Schmidt, and Shultz (2008) report an average correlation of .44 between extraversion and PA. There is also a clear link between neuroticism and negative affect (NA). Those scoring highly on anxiety and low on emotional stability experience negative emotional states more often compared to people scoring low on anxiety and high on stability (Yik \& Russell, 2001; Miller, Vachon, \& Lynam, 2009). In a meta-analysis of 73 studies, Steel et al. (2008) found an average correlation of .54 between neuroticism and NA.

The relationships described above are based on correlational studies, and no causal links can be derived from them. However, most theoretical accounts view personality as causally antecedent to affectivity. For example, Eysenck and Eysenck (1985) as well as Costa and McCrae (1980) propose that extraversion and neuroticism predispose individuals to experience more positive and negative affect, respectively. Wilt et al. (2012) theorized that trait extraversion increases the propensity to enact extraverted states, which in turn leads to experiencing more PA states. Empirical findings from experimental studies are limited and inconclusive. McNiel and Fleeson (2006) instructed participants to act more extraverted or more introverted during a discussion. The results indicated that subjects instructed to act extraverted exhibited more positive affect compared to the other group. A similar pattern applies to neuroticism and negative affect. Conversely, Rusting and Larsen (1997) found that by manipulating the mood one could influence the level of extraversion. Other studies also showed that ex- traverted behaviors could result from pleasant mood induction (Cunningham, 1988) but also that extraverts show heightened emotional reactivity to positive mood induction (Larsen \& Ketelaar, 1991) and greater affective reactivity in response to appetitive stimuli (Smillie, Cooper, Wilt, \& Revelle, 2012).

Individual differences in personality and the associated differences in affectivity could be the result of the underlying mechanisms related to reward and punishment sensitivity (Gross, Sutton, \& Ketelaar, 1998). Gray's Reinforcement Sensitivity Theory (RST; Gray, 1982; Gray \& McNaughton, 2000) proposes the existence of several neural networks related to reward sensitivity (BAS - behavioral activation system) and punishment sensitivity (BIS - behavioral inhibition system; FFFS - fight, flight and freeze system). Individual differences in behavior could reflect different levels of reactivity of these neural networks. Punishment and reward sensitivities can be seen as the biological foundation for the surface representation of extraversion and neuroticism (Corr, 2004).

The behavioral activation system is triggered in potentially rewarding situations. Individuals with a more sensitive BAS should react more strongly to rewards and should approach them more readily. The behavioral inhibition system is triggered in potentially threatening situations or in cases of approach-avoidance conflict. Individuals with a more sensitive BIS should react more strongly to punishment and should be more cautious when approaching potentially threatening situations.

The theory relates sensitivity of the BIS and BAS systems to extraversion and neuroticism. The predictions vary somewhat between the first (Gray, 1982) and second (Gray \& McNaughton, 2000) version of the theory, but some links are expected in both cases. Because social interactions are often rewarding, high BAS individuals will pursue more social interactions and will thus behave in a more extroverted manner. On the other hand, the proposed function of the BIS system is to inhibit behavior and induce the feeling of anxiety in situations of potential danger, which overlaps with the definition of neuroticism. Empirical data support both links. Keiser and Ross (2011), for example, report a correlation of .47 between BAS sensitivity and extraversion and .51 between BIS sensitivity and neuroticism.

Elliot and Thrash (2002) used a confirmatory factor analytic approach to test the structural validity of all three sets of constructs described above. Even though they had no way of postulating the causal relationship, they concluded that there is an underlying common cause for BAS sensitivity, extraversion and PA, which they termed approach temperament. Furthermore, they postulated the existence of a common cause for BIS sensitivity, neuroticism and NA, which they termed avoidance temperament. We will use these labels to represent the "shared meaning" of 
the positive and negative constructs described above. Larsen and Augustine (2008) highlight the same relationships and draw the following conclusion: "Broad links between the personality traits of extraversion, positive affectivity, and the behavioral activation system suggest an underlying dispositional tendency to approach. Additionally, broad links between the personality traits of neuroticism, negative affectivity, and the behavioral inhibition system suggest an underlying dispositional tendency to avoid." (2008; p. 161).

This study aims to provide indirect support for some of the predictions based on Gray's RST (1982; Gray \& McNaughton, 2000) and the work of Costa and McCrae (1980) and Eysenck and Eysenck (1985) with respect to the relationship between BIS/BAS sensitivity, personality and affectivity. We hypothesized that the links between these constructs will reflect the causal direction derived from the aforementioned theories: BIS/BAS sensitivity will precede personality (extraversion/neuroticism), which will in turn precede affectivity (PA/NA). The data were collected at two time points to allow for testing the stability and cross-lagged effects. We compared two sets of models using latent variables representing the constructs in question. One set of models represented approach temperament and included BAS, extraversion and trait positive affect, while the other set represented avoidance temperament and included BIS, neuroticism and trait negative affect. For both sets of constructs, a baseline stability model was specified that included only paths between the same variables across the two time points. A directional model that included paths according to the theoretical predictions described above was tested against the baseline. Furthermore, a complete cross-lagged model was also tested against the stability one, to allow for potential influences not predicted based on our theoretical perspective. If BIS/BAS sensitivity does indeed manifest itself like neuroticism/extraversion, and if these do in turn predispose individuals to experience more negative/positive emotions, we expect the directional model to have the best fit to the data, improving it significantly over the baseline.

\section{PARTICIPANTS AND PROCEDURE}

\section{PARTICIPANTS}

A convenience sample of 286 participants (53 males, 233 females), students from the Faculty of Humanities and Social Sciences at the University of Zagreb participated in the study for course credit or financial reimbursement. The mean age of the participants was 21 years, with a range from 18 to 32 and a standard deviation of 1.90 . Of the total number, 270 had valid results on all instruments at both time points. The interval between the two time points was approximately four weeks (30 days).

\section{INSTRUMENTS}

BIS/BAS sensitivities from Gray's reinforcement sensitivity theory were measured by the Croatian translation of the BIS/BAS scales (Carver \& White, 1994; Križanić, Greblo, \& Knezović, 2015; Rebernjak \& Buško, 2015). This instrument is based on the first version of Gray's theory and hence includes a joint BIS and FFFS sensitivity measure. It uses a three-dimensional approach to BAS, measuring separately drive (BASD), reward responsiveness (BASR) and fun (BASF). On the second level of the hierarchy, these three dimensions form an underlying BAS factor. We followed this unidimensional conceptualization of BAS in specifying our models. Cronbach's $\alpha$ of the BIS scale was .81 at the first and .82 at the second time point, while test-retest reliability was estimated at .86. For the BAS scale $\alpha$ was .75 at the first and .76 at the second time point, while test-retest reliability was estimated at .78.

Extraversion and neuroticism were measured by the 20-item version of the Croatian translation of the IPIP scale (Goldberg, 1999; Mlačić \& Goldberg, 2007). Internal consistency proved adequate, as Cronbach's $\alpha$ for extraversion was estimated to be .93 at both time points, while test-retest reliability was estimated at .94. Internal consistency for neuroticism was .92 at the first and .94 at the second time point, while test-retest reliability was estimated at .92 . The results of exploratory factor analyses suggest a clear one-factor solution for both constructs.

Positive and negative affect were measured by a Croatian translation of the trait version of the PANAS scale (Watson, Clark, \& Tellegen, 1988; Križanić \& Knezović, 2007). The participants were asked to assess the extent to which they have felt certain emotions during the past few weeks. Cronbach's $\alpha$ of the $P A$ subscale was .82 at the first and .85 at the second time point, while test-retest reliability was estimated at .63. Estimates were similar for the $N A$ subscale $-\alpha$ was .84 and .90 for the two time points, respectively, while test-retest reliability was .68. Again, a clear one-factor solution was obtained for both PA and NA at both time points.

\section{MODEL SPECIFICATION AND ANALYSIS}

For both approach and avoidance temperament we specified a set of models reflecting specific hypotheses about the relationships among the three constructs. Simple stability models served as the baseline in both cases. These models contained only the paths between the same construct at two time points. In other words, only auto-correlations or stability coefficients were included. For each case, two models were specified to be compared to the stability one: a directional model and a full cross-lagged model. In 
the directional model three direct paths were introduced, implying causal direction from reinforcement sensitivity to personality and affectivity as well as from personality to affectivity. The full cross-lagged model was specified with all six cross-lagged effects. Based on the results of these analyses, the final best fitting model was chosen and re-specified with all the insignificant parameters set to zero.

All the models were specified using parcels as indicators. Parcels for extraversion/neuroticism, PA/NA and BIS were constructed by averaging over half of the items for the first parcel, and the other half for the second. BAS was defined using total scores on the three subscales (BASD, BASR, BASF) as indicators. This is what caused the difference in the degrees of freedom between the models for approach and avoidance temperaments.

Factor saturations for the equivalent manifest variables were constrained to be equal across the two time points, ensuring weak factorial invariance. Covariances between the error terms of the equivalent manifest variables were estimated freely across the two measurement occasions, allowing for method effects.

To evaluate model fit we used the following criteria: A good fitting model was characterized by an insignificant $\chi^{2}$ test (minimum fit function $\chi^{2}$ ) and an RMSEA under .05. The fit of the nested models within a set (approach or avoidance temperament) was tested using the $\chi^{2}$ difference test. The analyses were done using the LISREL software (Jöreskog \& Sörbom, 2004).

\section{RESULTS}

Descriptive statistics and intercorrelations between the indicators used are omitted from this report due to the size of the tables but are available upon request. Summary data and model comparisons for the two sets of models representing approach and avoidance temperament are given in Table 1. Standardized factor saturations for all indicators used in all models and at both time points were estimated at values greater than 0.80 , with the exception of indicators measuring BAS, where the estimates ranged from 0.30 to 0.51 at the first, and 0.35 to 0.60 at the second time point.

The stability model in the case of the constructs describing approach temperament (S1) showed a good fit to the data. Extraversion and BAS proved very stable across the one-month period (.97 and .94, respectively), while PA exhibited somewhat lower stability (.67). The directional model (D1) showed a better fit to the data compared to S1. Only one of the three paths introduced in the model was significantly different from zero - the link between extraversion at $\mathrm{T} 1$ and positive affect at $\mathrm{T} 2$. The full cross-lagged model (C1) also showed a significant increase in fit compared to S1, with both paths between extraversion and positive affect significantly differing from zero. The $\mathrm{C} 1$ model was then re-specified such that insignificant paths were fixed to 0 . The structural part of this final model (F1) for approach temperament is presented in Figure 1.

In the case of the constructs describing avoidance temperament the stability model (S2) showed adequate fit to the data. Neuroticism and behavioral inhibition sensitivity showed again higher stability estimates (.95 and .96 respectively) compared to NA (.75). The directional model (D2) was in this case also a significant improvement over S2, with the path between BIS at $\mathrm{T} 1$ and neuroticism at $\mathrm{T} 2$ being the only significant causal link. The full cross-lagged model (C2) showed no significant improvement over the baseline (S2). The final model (F2) was re-specified from D2 by fixing all the insignificant paths to zero. The structural part of F2 for avoidance temperament is presented in Figure 2.

In summary, our data show that a well-fitting model can be specified both for approach and avoid-

Table 1

Some indicators of model fit for the sets of nested models for approach and avoidance temperament

\begin{tabular}{lcccccccc}
\hline Model & $\chi^{2}(d f)$ & $p\left(\chi^{2}\right)$ & $\chi^{2} / d f$ & RMSEA & Comparison & $\Delta\left(\chi^{2}\right)$ & $\Delta(d f)$ & $p(\Delta)$ \\
\hline Approach temperament & & & & & & & & - \\
$\quad$ Stability (S1) & $84.76(65)$ & .050 & 1.30 & .035 & - & - & - & - \\
$\quad$ Directional (D1) & $74.26(62)$ & .137 & 1.19 & .028 & S1-D1 & 10.50 & 3 & .015 \\
Full cross-lagged (C1) & $68.37(59)$ & .190 & 1.16 & .025 & S1-C1 & 16.39 & 6 & .012 \\
Final (F1) & $71.16(63)$ & .230 & 1.13 & .022 & & & \\
Avoidance temperament & & & & & & & - \\
$\quad$ Stability (S2) & $65.27(42)$ & .012 & 1.55 & .043 & - & - & - & -040 \\
Directional (D2) & $56.94(39)$ & .032 & 1.46 & .039 & S2-D2 & 8.33 & 3 \\
Full cross-lagged (C2) & $55.01(36)$ & .022 & 1.53 & .042 & S2-C2 & 10.26 & 6 & .114 \\
Final (F2) & $60.90(41)$ & .023 & 1.49 & .041 & & & \\
\hline
\end{tabular}




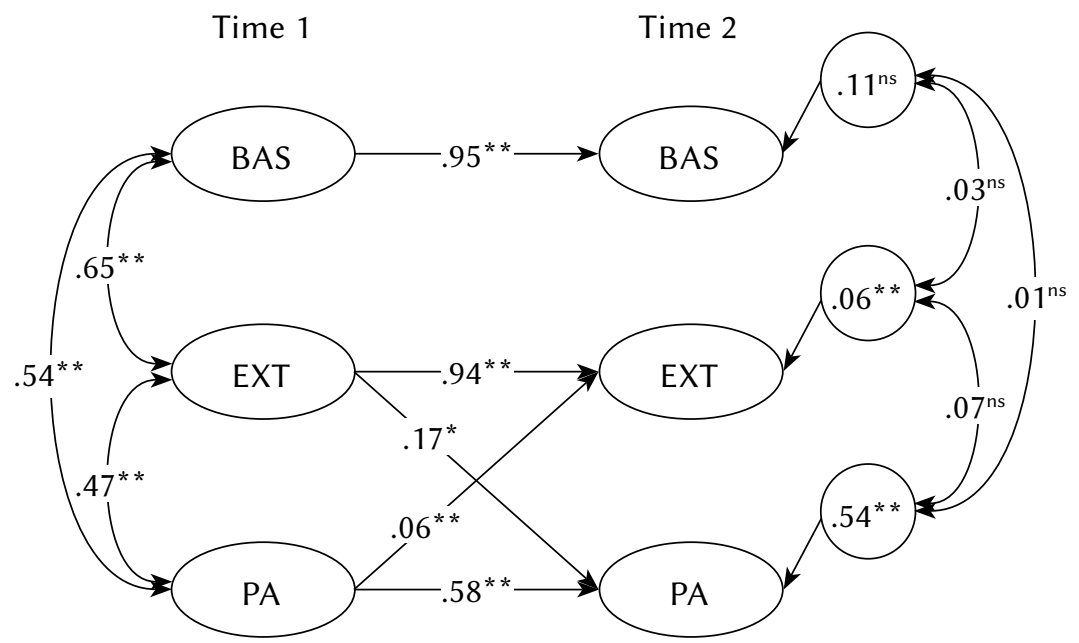

Note. BAS - behavioral activation system, EXT - extraversion, PA - positive affect.

Figure 1. The structural part of the final (F1) model for approach temperament. All path coefficients are standardized. Disturbances are depicted as the percentage of variance not explained by the variables in the model.

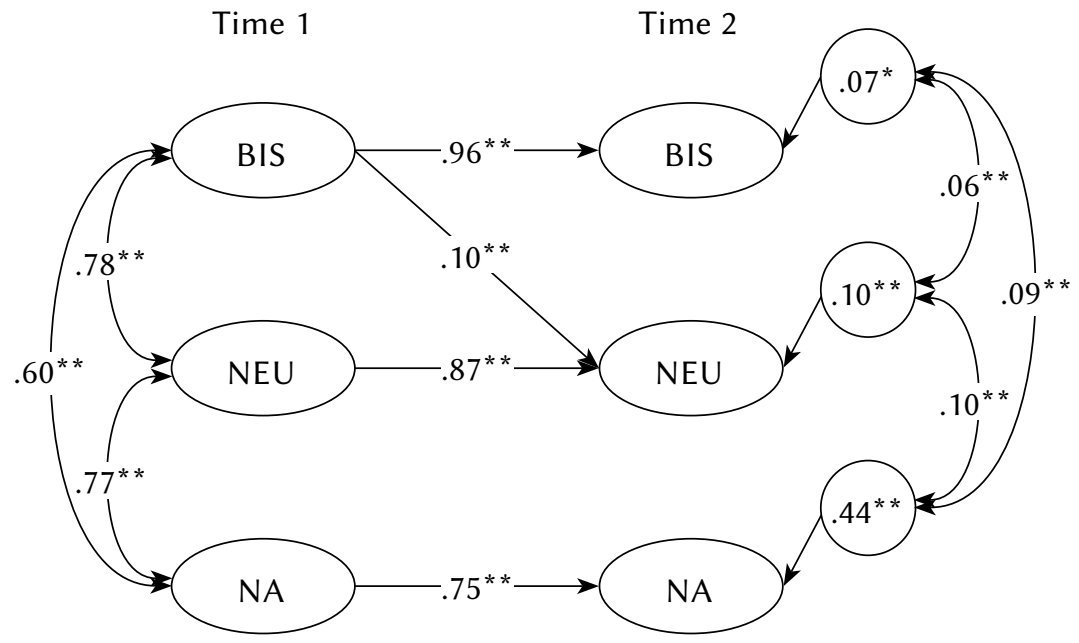

Note. BIS - behavioral inhibition system, NEU - neuroticism, NA - negative affect.

Figure 2. The structural part of the final (F2) model for avoidance temperament. All path coefficients are standardized. Disturbances are depicted as the percentage of variance not explained by the variables in the model.

ance temperament. In both cases, the best models (F1 and F2) are those that allow for coefficients between different constructs to be estimated freely across the two occasions. In the case of approach temperament, the links between PA and extraversion were significantly different from zero in both directions, implying a reciprocal relationship. In the case of avoidance temperament, the only significant link besides the stability estimates was the one between BIS at T1 and neuroticism at $\mathrm{T} 2$.

\section{DISCUSSION}

The study examined the relationships within two sets of affective constructs: personality, affectivity and re- inforcement sensitivity. More specifically, we tested on the one hand the hypothesized direction of the relationships between behavioral activation sensitivity, extraversion and positive affectivity, i.e. constructs representing approach temperament, as they share the propensity to approach potential rewards. On the other hand, we tested the direction of the relationships between behavioral inhibition sensitivity, neuroticism and negative affectivity, portraying avoidance temperament, as they share the propensity to avoid potential threats. Following theoretical accounts such as the RST (Gray, 1987), we expected the BIS/BAS sensitivities to be antecedent to personality, with personality in turn predicting affectivity. The hypotheses translated into a series of structural equations were tested by SEM methodology. 
Both sets of stability models (S1 and S2) showed adequate fit to the data, indicating that even without any links between different constructs, the models describe the observed data well. High stability coefficients are obvious in both approach and avoidance temperament models for behavioral activation/inhibition sensitivity and personality measures (extraversion and neuroticism). These results are in line with theoretical expectations on personality and reinforcement sensitivity as constructs stable in time (McCrae \& Costa, 1994; Gray \& McNaughton, 2000).

Measures of positive and negative affect exhibited lower stability compared to behavioral activation/ inhibition sensitivities and personality. Even though PANAS was used to assess trait affectivity, the lower estimates of stability of this measure suggest the existence of contextual influences, as stability was significantly lower than the measures of internal consistencies at the two time points. Lower estimates of stability for affectivity when compared to BIS/BAS and $\mathrm{E} / \mathrm{N}$ were expected based on previous research. The stability estimates for PA and NA observed in our study are lower than the ones obtained by Watson et al. (1988; .68 and .71 for PA and NA, respectively) and Thompson (2007; .84 for both PA and NA). Both of these studies used an 8-week period between test and retest, compared to 4 weeks in our case.

Allowing the directional effects in accordance with the theoretical accounts significantly improved the fit for both sets of models (D1 and D2). In other words, allowing the behavioral activation/inhibition sensitivities at T1 to predict personality and affectivity at $\mathrm{T} 2$, as well as allowing extraversion/neuroticism to predict affectivity at $\mathrm{T} 2$, resulted in models with significantly better fit to the data compared with the stability ones. This supports the plausibility of the theoretical accounts by Gray $(1982,1987)$ and Costa and McCrae (1980) with respect to the causality between the stable affective constructs. A full crosslagged model was a significant improvement over the stability model only in the case of approach temperament. Both final models show a good fit to the data.

The structural part of the final model for approach temperament (Figure 1) shows no impact of BAS sensitivity on extraversion or PA across time points. In other words, when controlling for the stability effects, BAS at T1 has no significant contribution to explaining PA or extraversion variances at T2. This finding is unexpected, since BAS was explicitly defined as an underlying cause of extraversion (Gray, 1987), and other authors have previously hinted at this expected causal direction (Smits \& Boeck, 2006). It is possible that the chosen period between measurements was not adequate in establishing this relationship. Furthermore, reward responsiveness might be an important determining factor of extraversion at the time the personality is forming, but might cease to be so in young adults.
The same model (Figure 1) shows a complex relationship between personality and affect: a reciprocal pattern of relationships was observed for extraversion and positive affectivity across measurement occasions. When controlling for stability effects, extraversion at $\mathrm{T} 1$ predicts $\mathrm{PA}$ at $\mathrm{T} 2$, but also PA at $\mathrm{T} 1$ predicts extraversion at $\mathrm{T} 2$. One explanation of this relationship is that people who score higher on measures of extraversion tend to enact more extraverted states (Wilt et al., 2012), and it is these extraverted states that are related to the positive affect. Positive affect can, in turn, produce more extraverted states - which was manifested here as a causal link between PA at T1 and extraversion at T2. Furthermore, this finding is in line with both causal directions between extraversion and PA described in the introduction (McNiel \& Fleeson, 2006; Rusting \& Larsen, 1997).

In the case of avoidance temperament (Figure 2) there is a significant link between the sensitivity of BIS at T1 and neuroticism at T2. Even though BIS and neuroticism constructs overlap to a large degree, our results seem to suggest that BIS sensitivity can predict neuroticism after a period of 4 weeks even after controlling for neuroticism at T1. This finding is in line with expectations drawn from RST, namely that neuroticism or anxiety can be seen as surface manifestations of BIS sensitivity (Gray, 1987; Gray $\&$ McNaughton, 2000). Furthermore, neuroticism and NA share no significant causal links between the two measurement occasions after controlling for stability effects. This is at odds with the same part of the $a p$ proach temperament model, which showed a reciprocal causal link between extraversion and PA.

In conclusion, the results presented here give limited support to the proposed direction of relationships between the three sets of affective constructs investigated in this study. It is a well-established fact that BAS sensitivity, extraversion and PA on the one hand, and BIS sensitivity, neuroticism and NA on the other, are closely related constructs (Larsen \& Augustine, 2008; Elliot \& Thrash, 2002). However, disentangling their effects from the conceptual overlaps is no easy thing. Our results suggest that, even after controlling for stability effects, there are some direct links across two measurement occasions. On the one hand, as predicted by RST, a weak but significant path was found between BIS sensitivity at T1 and neuroticism at T2; however, there were no other significant links between the constructs of avoidance temperament. On the other hand, a complex relationship between extraversion and positive affectivity was highlighted with the reciprocal causal link between these two constructs.

Some limitations of the present study are worth mentioning. First, our sample was homogeneous with regard to age, sex and educational background, which severely restricts the ability to generalize our 
results. Second, some of the instruments employed, though widely used, have severe limitations. The BIS/ BAS questionnaire measures the constructs from the outdated version of Gray's RST, and confounds BIS and FFF sensitivities. Results derived from PANAS make it hard to disentangle long-term affective traits from mood states, even when the trait version was employed. Third, only two measurement occasions were used in this study, and the results should be replicated in a study designed to take into consideration more time points. Future research attempts should be made in distinguishing state from trait effects for both personality and affect constructs, as well as reinforcement sensitivity. It is possible that the complex relationships between these constructs reflect the confusion from confounding state and trait effects.

\section{RefERENCES}

Carver, C. S., \& White, T. L. (1994). Behavioral inhibition, behavioral activation, and affective responses to impending reward and punishment: The BIS/BAS Scales. Journal of Personality and Social Psychology, 67, 319.

Corr, P. J. (2004). Reinforcement sensitivity theory and personality. Neuroscience \& Biobehavioral Reviews, 28, 317-332.

Costa, P. T., \& McCrae, R. R. (1980). Influence of extraversion and neuroticism on subjective well-being: happy and unhappy people. Journal of Personality and Social Psychology, 38, 668-678.

Cunningham, M. R. (1988). What do you do when you're happy or blue? Mood, expectancies, and behavioral interest. Motivation and Emotion, 12, 309-331.

Elliot, A. J., \& Thrash, T. M. (2002). Approach-avoidance motivation in personality: approach and avoidance temperaments and goals. Journal of Personality and Social Psychology, 82, 804-818.

Eysenck, H. J., \& Eysenck, M. W. (1985). Personality and Individual Differences. New York: Plenum.

Gable, S. L., Reis, H. T., \& Elliot, A. J. (2003). Evidence for bivariate systems: An empirical test of appetition and aversion across domains. Journal of $R e-$ search in Personality, 37, 349-372.

Goldberg, L. R. (1999). A broad-bandwidth, public domain, personality inventory measuring the lower-level facets of several five-factor models. In I. Mervielde, I. Deary, F. De Fruyt, \& F. Ostendorf (eds.), Personality Psychology in Europe (vol. 7, pp. 7-28). Tilburg, The Netherlands: Tilburg University Press.

Gray, J. A. (1982). The neuropsychology of anxiety: an enquiry into the functions of the septo-hippocampal system. Oxford: Oxford University Press.

Gray, J. A. (1987). The psychology of fear and stress. Cambridge: Cambridge University Press.
Gray, J. A., \& McNaughton, N. (2000). The Neuropsychology of anxiety: An enquiry into the functions of the septo-hippocampal system ( $2^{\text {nd }}$ ed.). Oxford: Oxford University Press.

Gross, J. J., Sutton, S. K., \& Ketelaar, T. (1998). Affective-reactivity views. Personality and Social Psychology Bulletin, 24, 279.

Jöreskog, K. G., \& Sörbom, D. (2004). LISREL 8.7 for Windows [Computer software]. Lincolnwood, IL: Scientific Software International, Inc.

Keiser, H. N., \& Ross, S. R. (2011). Carver and White's BIS/FFS/BAS scales and domains and facets of the five factor model of personality. Personality and Individual Differences, 51, 39-44.

Križanić, V., Greblo, Z., \& Knezović, Z. (2015). Measures of Behavioral Inhibition and Activation System Sensitivity as Predictors of Big Five Personality Traits. Psihologijske teme, 24, 305-324.

Križanić, V., \& Knezović, Z. (2007). Some methodological aspects of PANAS-X scale adaptation. In G. Keresteš, I. Brković, \& A. Butković (eds.), 18. Ramiro and Zoran Bujas Days - book of abstracts (pp. 106). Zagreb, Croatia: Grafički zavod Hrvatske.

Larsen, R. J., \& Augustine, A. A. (2008). Basic personality dispositions related to approach and avoidance: Extraversion/neuroticism, BAS/BIS, and positive/negative affectivity. In A. J. Elliot (ed.), Handbook of Approach and Avoidance Motivation (pp. 151-164). New Jersey: Erlbaum.

Larsen, R. J., \& Ketelaar, T. (1991). Personality and susceptibility to positive and negative emotional states. Journal of Personality and Social Psychology, 61, 132-140.

McCrae, R. R., \& Costa, P. T. (1994). The stability of personality: Observations and evaluations. Current Directions in Psychological Science, 3, 173-175.

McNiel, J. M., \& Fleeson, W. (2006). The causal effects of extraversion on positive affect and neuroticism on negative affect: Manipulating state extraversion and state neuroticism in an experimental approach. Journal of Research in Personality, 40, 529-550.

Miller, D. J., Vachon, D. D., \& Lynam, D. R. (2009). Neuroticism, negative affect, and negative affect instability: Establishing convergent and discriminant validity using ecological momentary assessment. Personality and Individual Differences, 47, 873-877.

Mlačić, B., \& Goldberg, L. R. (2007). An analysis of a cross-cultural personality inventory: The IPIP Big-Five factor markers in Croatia. Journal of Personality Assessment, 88, 168-177.

Rebernjak, B., \& Buško, V. (2015). An empirical evaluation of the measures of Gray's reinforcement sensitivity constructs. Suvremena psihologija, 18, 61-78.

Rusting, C. L., \& Larsen, R. J. (1997). Extraversion, neuroticism, and susceptibility to positive and 
negative affect: A test of two theoretical models. Personality and Individual Differences, 22, 607-612.

Smillie, L. D., Cooper, A. J., Wilt, J., \& Revelle, W. (2012). Do extraverts get more bang for the buck? Refining the affective-reactivity hypothesis of extraversion. Journal of Personality and Social Psychology, 103, 306-326.

Smits, D. J. M., \& De Boeck, P. (2006). From BIS/BAS to the Big Five. European Journal of Personality, 20, 255-270.

Steel, P., Schmidt, J., \& Shultz, J. (2008). Refining the relationship between personality and subjective well-being. Psychological Bulletin, 134, 138-161.

Thompson, E. R. (2007). Development and validation of an internationally reliable short-form of the positive and negative affect schedule (PANAS). Journal of Cross-Cultural Psychology, 38, 227-242.

Watson, D. (2000). Mood and temperament. New York: Guilford Press.

Watson, D., \& Clark, L. A. (1997). Extraversion and its positive emotional core. In R. Hogan, J. A. Johnson, \& S. R. Briggs (eds.), Handbook of Personality Psychology (pp. 767-793). San Diego, CA: Academic Press.

Watson, D., Clark, L. A., \& Tellegen, A. (1988). Development and validation of brief measures of positive and negative affect: the PANAS scales. Journal of Personality and Social Psychology, 54, 1063-1070.

Wilt, J., Noftle, E. E., Fleeson, W., \& Spain, J. S. (2012). The dynamic role of personality states in mediating the relationship between extraversion and positive affect. Journal of Personality, 80, 1205-1236.

Yik, M. S. M., \& Russell, J. A. (2001). Predicting the big two of affect from the big five of personality. Journal of Research in Personality, 35, 247-277. 